\title{
Positioning Methods and Technologies in Mobile and Pervasive Computing
}

\author{
Dragan Stojanovic \\ University of Nis, Serbia \\ Billur Barshan \\ Bilkent University, Ankara, Turkey \\ Apostolos Papadopoulos \\ Aristotle University of Thessaloniki, Greece \\ Nico Van de Weghe \\ Ghent University, Belgium \\ Christophe Claramunt \\ Naval Academy Research Institute, Brest, France
}

\section{BACKGROUND}

The core of any positioning method relies in the realtime measurement of one to several parameters, such as angles, distances, or distance differences (Hightower $\&$ Borriello, 2001). Measurement parameters reflect the location of a target object relative to a single point or several fixed points in the environment with known locations. Such parameters are measured using the physical characteristics of electromagnetic radio and infrared signals, as well as ultrasound signals, such as their travel time, velocity or attenuation. After the determination of the required parameters, the target object's location can be calculated using measurement results and the known locations of the fixed points.

There are four principal techniques and methods for location calculation and estimation:

- $\quad$ The proximity technique (Figure 1a) derives the location of a target object with respect to its vicinity to the location of known object(s). A target object receives the signal from a given node, so the location of the node or the cell identification defines the location of a target.

DOI: $10.4018 / 978-1-4666-5888-2 . c h 564$ 
- The triangulation technique uses the triangle geometry to compute locations of a target object. It is applied via lateration (actually trilateration) (Figure 1b), that uses distance measurements to points with known locations, or via angulation (sometimes also referred as triangulation) (Figure 1c), which measures angles relative to points with known arrangement. Since electromagnetic/ultrasound signals move with known and nearly constant speed, determination of the time difference between sending and receiving a signal enable computation of the spatial distance between a transmitter and a receiver. Known distances from three or more transmitters provide accurate positioning of the target object. For the angulation technique, antennas with direction capabilities are used. Given two or more directions from fixed locations to the same object, the location of the target object can be computed.

- Scene analysis techniques involve examination and matching a video/image or electromagnetic characteristics viewed/sensed from a target object. Analysis of electromagnetic "scene" sensed by a target object defined by electromagnetic signals and their strengths from different transmitters, provide the determination of location using a pattern matching, radio map technique. Using video cameras, a positioning system can detect significant patterns in a video data stream to determine the user's location. If users wear badges with certain labels, they can be detected in video images. At the other extreme are techniques involving the matching of perspective video images of the environment to 3D models stored in an image/video database.

- Dead reckoning techniques provide estimation of the location of a target object based on the last known location, assuming that the direction of motion and either the velocity of the target object or the traveled distance are known.

\section{POSITIONING TECHNOLOGIES, SYSTEMS AND APPLICATIONS}

Positioning technologies and systems based on one or more positioning techniques and methods possess different characteristics that determine their suitability for specific LBS applications.

\section{Characteristics and Classification of Positioning Technologies}

Positioning systems can be based on either an existing communication network, or a dedicated network/ infrastructure that is only used to receive/transmit positioning signals. Mobile devices can be active when transmitting a signal themselves, or passive when just receiving a signal. The location can be determined in the mobile device itself, in the wireless network, or within dedicated positioning infrastructure.

Positioning systems differ in accuracy, precision, scope, the type of determined location: geometric or symbolic, and the cost. An estimated location is considered accurate if it corresponds, as much as possible, to the true location of a target object. Precision refers to the repeatability of the measurement and indicates how sharply a location can be defined for the sequence of

Figure 1. Location sensing techniques: a) Proximity; b) Trilateration; c) Angulation
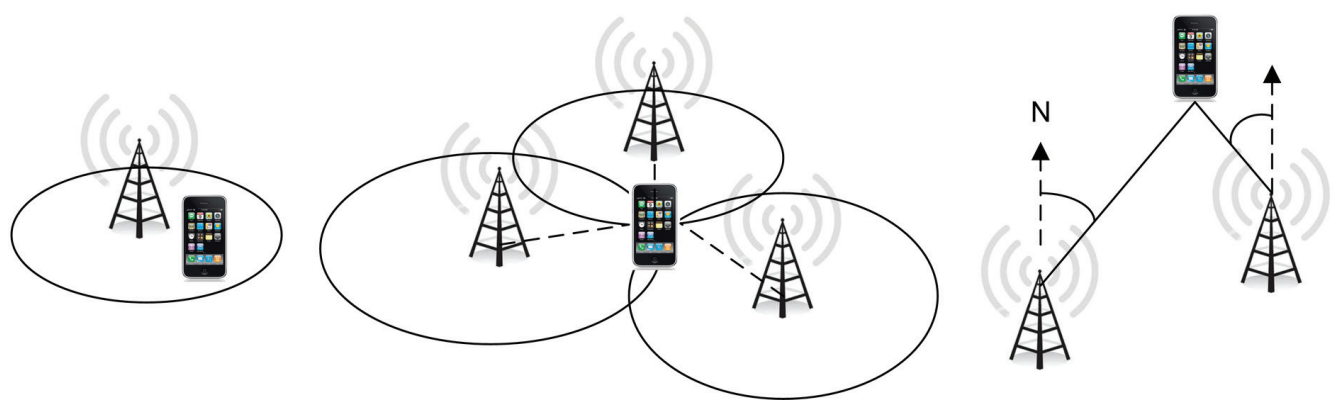
location determinations. The accuracy of a positioning system could be defined by an uncertainty area, i.e. the location is actually defined as an ellipse (ellipsoid) around the determined location.

A positioning system delivers a target object's location with regard to a spatial reference system or to a defined symbolic space. Geometric location is determined in defined geodetic/cartographic reference system, such as WGS-84, UTM, or a local coordinate system. Some positioning systems determine symbolic locations in terms of cell identifiers, room numbers, buildings, places of interest, etc. Positioning systems differ also in scope of location determination. Since the majority of positioning methods depend on the propagation properties of electromagnetic signals their effectiveness and efficiency depend on the environment. Some positioning systems are intended for outdoor space with the scope that may be the whole Earth, a certain region or a metropolitan area. There exist a variety of positioning technologies and solutions intended for indoor spaces that require some kind of infrastructure that is usually not available in open space. A positioning system determines also the cost needed for a system to be fully operative, such as time and space for installation and maintenance of required infrastructure, as well as the price of equipment that is required.

Positioning systems and technologies can be classified according to the scope of infrastructure needed for positioning (satellite, cellular, WLAN and WPAN), the type of such infrastructure if any, their suitability for outdoor or indoor spaces, the part of the system where the location is calculated/estimated, i.e., whether a mobile device locates itself or is tracked. These characteristics highly determine the usability of particular positioning technologies and systems for specific LBS applications.

Thus, positioning technologies are classified as:

- Satellite positioning (GPS) is based on the satellite infrastructure with global scope, outdoor spaces and the location is determined by the mobile device (GPS receiver).

- Cellular network positioning is based on cellular network infrastructure, with scope of urban areas and outdoor space, and employs triangulation method that estimates the location of a mobile device at the infrastructure.
- WLAN and WPAN technologies (Wi-Fi, Bluetooth, UWB, Zigbee, RFID, infrared, ultrasound, etc.) are based on wireless communication or stand-alone infrastructure constrained to indoor and limited outdoor spaces, providing determination of location either at the mobile device or in the infrastructure.

- Dead reckoning positioning technologies uses motion sensors and odometers to determine location at the device without the need for infrastructure, with the scope limited by accumulated error.

- Video scene analysis represents technologies based on the processing of video signals to detect specific tags in the scene (barcodes) or match the scene with prerecorded images/video to determine the location at either the mobile device itself, or to track target objects moving in the scene.

\section{Pervasive Positioning Systems}

Many different positioning systems have been developed that provide appropriate location data to locationbased and context-aware services.

In open space (outdoors), mobile devices/users are most commonly and accurately positioned by GPS technology and satellite-based infrastructure that provides location as a point in a geodetic reference system. The main disadvantage of GPS-based positioning is its dependency on a direct line-of-sight with the satellites and thus unavailability in indoor spaces, as well as in natural/urban canyons. The current satellite positioning systems are: NAVSTAR Global Positioning System (http://www.gps.gov/), GLONASS (http:// www.glonass-ianc.rsa.ru/en/) and Galileo (http://www. esa.int/esaNA/galileo.html), which is expected to be fully operational in 2020 .

Cellular networks provide several positioning methods based on wireless communication infrastructure. The simplest one is the cell identification (Cell-ID) method that uses the proximity technique. The accuracy is low and depends on the size of the network cells. Other positioning methods employ trilateration and angulation techniques based on known locations of cellular base stations. TOA (Time of Arrival) method is based on measuring the travel time of a radio signal 
from a mobile device to a remote base station and the trilateration technique to estimate the location of the mobile device with an accuracy of 100 to $150 \mathrm{~m}$. Time Difference of Arrival (TDOA) method measures the time difference between signals travelling between the mobile device and two reference base stations. This determines the location of the mobile device on a curve between any two base stations used in positioning. The accuracy of TDOA (OTDOA, U-TDOA) is between 50 and $120 \mathrm{~m}$. The Angle of Arrival (AOA) determines the location of the mobile deice based on angulation technique with the accuracy between 100 and $150 \mathrm{~m}$. In the E-OTD (Enhanced Observed Time Difference) technique the receiver makes the TDOA measurements and either sends them back to the network for location calculation, or calculates the location. The accuracy of E-OTD is expected to be as good as $50-100 \mathrm{~m}$. The accuracy of cellular network positioning technologies is higher with the density of base stations in highly populated urban areas.

In indoor environments, positioning is mainly achieved through the use of radio technologies, such as Bluetooth, Zigbee, UWB, RFID, and Wi-Fi, or infrared and ultrasound technologies (Gu et al., 2009) (Lie et al., 2007). The most popular indoor positioning systems use Wi-Fi technology, because of wide availability of Wi-Fi network infrastructure. Wi-Fi positioning systems use a radio propagation model to determine the distance to the various access points and then triangulation techniques (TOA, TDOA) to estimate location of a mobile device. Multipath distortion and variability of Wi-Fi signal strength in time limit the accuracy of such techniques. Most currently available Wi-Fi positioning solutions are based on the scene analysis technique, called the fingerprinting technique. The method uses the RSSI (Received Signal Strength Indication) to measure the strength of signals received from specific access points at discrete locations in space. This radio map has to be built before the system is operational. The calculation of the location consists of measuring the RSSI from several access points and then attempting to match these measurements with the RSSI values of previously calibrated location points stored in a radio map database. Depending on the pre-built radio map, the accuracy of the fingerprinting technique is expected to be in the range of 1 to $10 \mathrm{~m}$. One of the first Wi-Fi positioning systems was RADAR (Bahl \& Padmanabhan, 2000), and recent systems are Ekahau RTLS (http://www.ekahau.com), Skyhook Wireless (http://www.skyhookwireless.com/) and Navizon (http://www.navizon.com/).

On the other hand, UWB communication technology has emerged and provides better positioning accuracy than Wi-Fi. However, the high cost of UWB equipment and network deployment results in its limited availability for positioning (Sahinoglu et al., 2008). It is suitable for high-precision real-time positioning using TOA, TDOA, and AOA. UWB signals are less sensitive to multipath distortion and environment than conventional RF-based positioning systems, so they can achieve high accuracy. To date, several UWB positioning systems have been implemented and deployed, such as the Ubisense system (http://www.ubisense.net) and PulsON system developed by Time Domain (http:// www.timedomain.com/).

Bluetooth is a wireless technology that can be used for localization and tracking, mainly indoors. Bluetooth positioning systems have similar working principles as the self-localization schemes of sensor networks. The operation principle of both types of systems is based on obtaining the range information to anchor devices or access points and exploring unknown device locations using various algorithms. The majority of the available research and commercial systems are based on trilateration using the RSSI for calculating distances between Bluetooth devices, although several reported systems have explored proximity, cell-based approach. The positioning accuracy of the system is $1-5 \mathrm{~m}$ and depends on the positioning technique used and deployed infrastructure of Bluetooth or sensor devices. Current examples of Bluetooth positioning systems are ZONITH Indoor Positioning Module (http://www. zonith.com) and TOPAZ (http://www.tadlys.co.il).

In general, RFID systems are designed so that the reader detects the vicinity of a tag and retrieves the data stored in that tag. Therefore, the absolute location of the tag is not known but the RFID system is aware that a tag is placed at a certain range that depends on the type of the system used, either active or passive RFID. Besides the proximity technique that provides symbolic location of tag according to a reader location, there are several methods for performing accurate positioning using active RFID technology. These methods employ techniques such as AOA, TDOA and RSSI. The examples of location sensing systems using the RFID technology are SpotON (Hightower et al., 2000) and LANDMARC (Ni et al. 2004). 
Dedicated positioning methods, commonly based on infrared and ultrasound technologies, provide a high degree of accuracy, but require expensive equipment limited to a small scale that usually have high installation and maintenance costs (Gu et al., 2009). In infrared (IR)-based systems, each tracked person wears a small infrared device that emits a unique pulse signal representing its unique identifier. The signals are detected by at least one particular IR sensor in the vicinity. A location server estimates IR device location by aggregating data obtained from fixed IR sensors deployed within the indoor environment. The Active Badge system (Want et al., 1992), as the first IR positioning system, works this way and provides symbolic location information at the room or smaller level depending on deployed IR sensor infrastructure. Ultrasound-based systems use an ultrasound time-offlight lateration technique to provide more accurate physical positioning than by using infrared signals and sensors. The prominent examples are Active Bat system and the Cricket indoor location system (Gu et al., 2009).

There are also passive positioning systems that do not require specialized infrastructure. Such systems sense naturally occurring signals or physical phenomena and are based on, for example, magnetic compasses sensing the Earth's magnetic field, inertial sensors measuring acceleration and heading of an object in motion, and vision systems sensing scene and its features or recognizing specific visual patterns (barcodes). Dead reckoning is the process of estimating the current location of a moving device using the location calculated at a previous time instant, and the velocity, speed or heading estimate till the current time instant. Inertial navigation systems (INS) rely on the dead reckoning technique and can be used for both indoor and outdoor positioning. They use gyroscopes that provide angular rate information and accelerometers providing velocity rate information to continuously calculate the location, orientation, and velocity of a moving entity without the need for external references. However, even very small errors in the rate information provided by inertial sensors cause an unbounded growth in the error of the integrated measurements, usually referred as the "drift error." INSs have been implemented in various domains, such as for vehicle navigation (Skog \& Handel, 2009) and mobile robot positioning (Barshan \& Durrant-Whyte, 1995).
Visual positioning systems use low cost 2D tags (e.g., barcodes) with encoded information that can be recorded and processed by a mobile device with a built-in camera. The symbolic location of a device is estimated by finding the tag's identifier and associated location in a deployment database, or by decoding the location information embedded in the tag itself (Laere et al., 2008). Positioning systems based on video scene analysis are based on computer vision technology to recognize tracked objects in video data. Easy Living by Microsoft Research provides one example of this approach (Brumitt et al., 2000) where a video surveillance system tracks moving objects recognized in the video scenes. Also, the mobile device can use video scene analysis to estimate its location by comparing a snapshot of a scene generated by itself with a number of pre-observed simplified images of the scene taken from different positions and perspectives.

The important characteristics of positioning systems and technologies are reviewed in Table 1.

\section{Applications of Positioning Methods and Technologies in LBS}

Location-based services deliver geo-referenced information and geo-processing power to mobile users in accordance with their past, current and predicted location, or to the locations of the moving/stationary objects of their interest. Depending on services' requirements and context of their usage, different positioning technologies and systems are used at the heart of such service. There are a lot of classifications and taxonomies of location-based services presented in the literature so far (Figueiras \& Frattas, 2010), and also new services continue to emerge. The locationbased requirements and applications of positioning technologies and methods in various LBSs are reviewed in Table 2 along with accuracy required by a particular service (very high, from 0-5m, high, from $5-50 \mathrm{~m}$, medium, from $50-250 \mathrm{~m}$, and low, greater than $250 \mathrm{~m}$ ).

\section{FUTURE RESEARCH DIRECTIONS}

There are a lot of open issues and future research directions in domain of positioning technologies and location data collection systems that should be explored for the development of the next generation of location-based applications and services. 


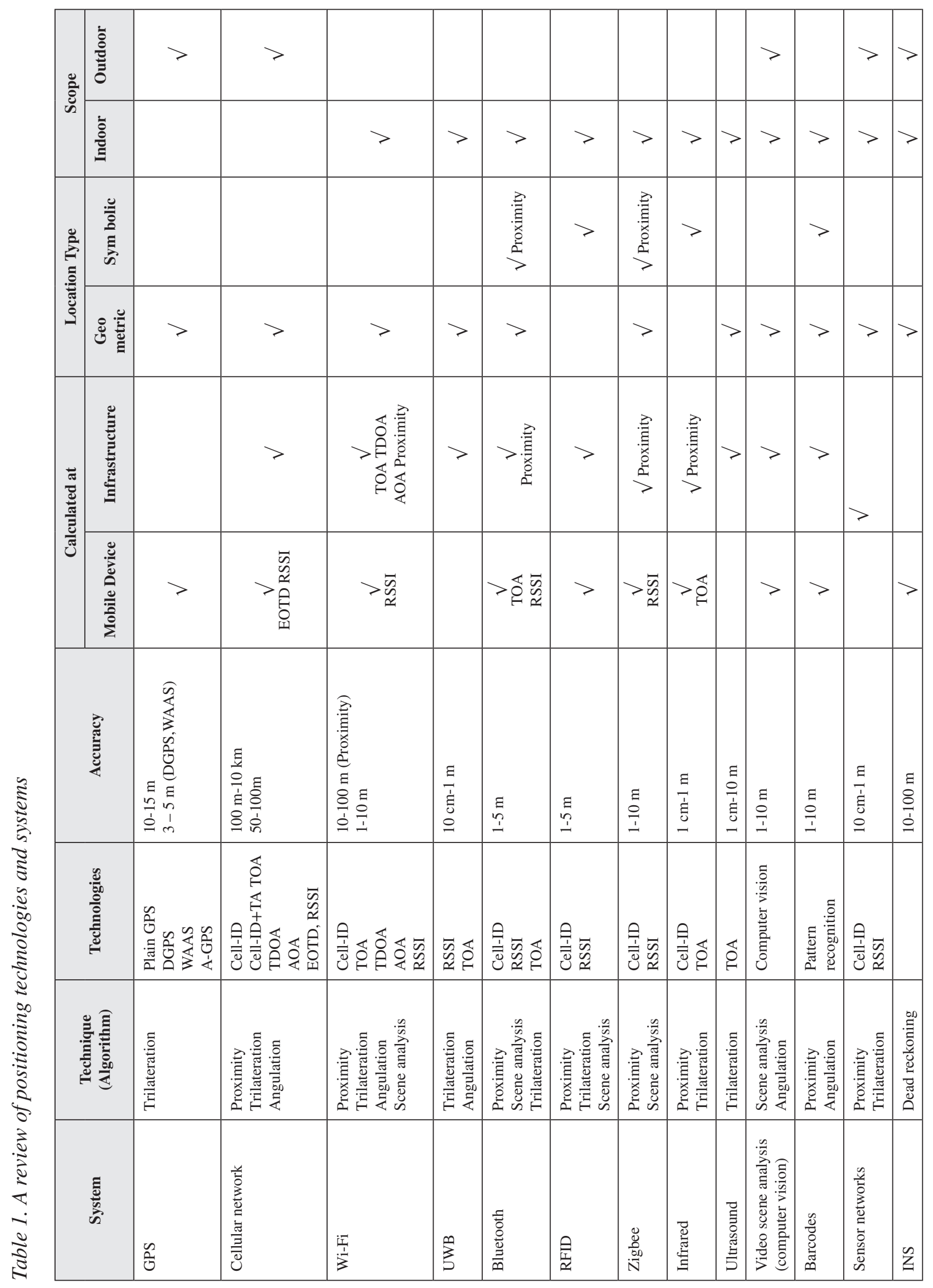


Table 2. Applications of positioning technologies and methods in various LBS domains

\begin{tabular}{|c|c|c|c|c|}
\hline $\begin{array}{l}\text { Application } \\
\text { Domain }\end{array}$ & Applications & $\begin{array}{l}\text { Positioning } \\
\text { Methods and } \\
\text { Technology }\end{array}$ & Accuracy & Examples \\
\hline $\begin{array}{l}\text { Information } \\
\text { services }\end{array}$ & $\begin{array}{l}\text { POI and Yellow pages } \\
\text { Tourist guide }\end{array}$ & $\begin{array}{l}\text { GPS, Cellular, } \\
\text { Wi-Fi }\end{array}$ & Medium & $\begin{array}{l}\text { Foursquare } \\
\text { (https://foursquare.com/) }\end{array}$ \\
\hline Traffic telematics & $\begin{array}{l}\text { Vehicle navigation } \\
\text { Traffic information } \\
\text { Roadside assistance }\end{array}$ & $\begin{array}{l}\text { GPS, Cellular, } \\
\text { Wi-Fi, RFID, } \\
\text { INS }\end{array}$ & $\begin{array}{l}\text { Medium to } \\
\text { high }\end{array}$ & $\begin{array}{l}\text { Waze } \\
\text { (http://www.waze.com) }\end{array}$ \\
\hline $\begin{array}{l}\text { Emergency, } \\
\text { safety, security }\end{array}$ & $\begin{array}{l}\text { Emergency call } \\
\text { Medical alert } \\
\text { Emergency management }\end{array}$ & $\begin{array}{l}\text { GPS, Cellular, } \\
\text { Wi-Fi, RFID, } \\
\text { Bluetooth }\end{array}$ & $\begin{array}{l}\text { Medium to } \\
\text { high }\end{array}$ & $\begin{array}{l}\text { Navizon - Applications in Health Care } \\
\text { (http://www.navizon.com/health-care) }\end{array}$ \\
\hline $\begin{array}{l}\text { Tracing and } \\
\text { tracking }\end{array}$ & $\begin{array}{l}\text { Elder care } \\
\text { Child tracking } \\
\text { Pets tracking } \\
\text { Asset tracking } \\
\text { Vehicle tracking }\end{array}$ & $\begin{array}{l}\text { GPS, Wi- } \\
\text { Fi, RFID, } \\
\text { Barcodes, INS }\end{array}$ & $\begin{array}{l}\text { High to very } \\
\text { high }\end{array}$ & $\begin{array}{l}\text { Tagg-The Pet Tracker } \\
\text { (http://www.pettracker.com/) }\end{array}$ \\
\hline $\begin{array}{l}\text { Personal } \\
\text { navigation }\end{array}$ & $\begin{array}{l}\text { Multi-modal transport } \\
\text { Trade fairs } \\
\text { Mass events } \\
\text { 3D indoor navigation }\end{array}$ & $\begin{array}{l}\text { GPS, Wi- } \\
\text { Fi, RFID, } \\
\text { Bluetooth }\end{array}$ & $\begin{array}{l}\text { Medium to } \\
\text { high }\end{array}$ & $\begin{array}{l}\text { In-Location Alliance } \\
\text { (http://in-location-alliance.com/) }\end{array}$ \\
\hline $\begin{array}{l}\text { Mobile resource } \\
\text { management }\end{array}$ & $\begin{array}{l}\text { Fleet management } \\
\text { Military force mgmt } \\
\text { Work force management }\end{array}$ & $\begin{array}{l}\text { GPS, RFID, } \\
\text { Sensor } \\
\text { networks, INS }\end{array}$ & $\begin{array}{l}\text { High to very } \\
\text { high }\end{array}$ & $\begin{array}{l}\text { TerSec - Emergency services solutions (http:// } \\
\text { www.tersec.eu/emergency-services-solutions. } \\
\text { php) }\end{array}$ \\
\hline $\begin{array}{l}\text { Community and } \\
\text { entertainment }\end{array}$ & $\begin{array}{l}\text { Mobile games } \\
\text { Entertainment services } \\
\text { Mobile social networking } \\
\text { Friend finder } \\
\text { Augmented reality }\end{array}$ & $\begin{array}{l}\text { GPS, Cellular, } \\
\text { Wi-Fi }\end{array}$ & $\begin{array}{l}\text { Medium to } \\
\text { high }\end{array}$ & $\begin{array}{l}\text { Google Latitude - geo-social network } \\
\text { (http://www.google.com/latitude) }\end{array}$ \\
\hline $\begin{array}{l}\text { Location-based } \\
\text { Billing }\end{array}$ & $\begin{array}{l}\text { Toll collection } \\
\text { Parking services }\end{array}$ & $\begin{array}{l}\text { GPS, } \\
\text { Bluetooth, } \\
\text { RFID, } \\
\text { Barcodes }\end{array}$ & High & $\begin{array}{l}\text { PayBySky - automated parking } \\
\text { (http://paybysky.com/) }\end{array}$ \\
\hline Commerce & $\begin{array}{l}\text { Marketing/advertisement } \\
\text { Mobile purchasing } \\
\text { Ticket selling }\end{array}$ & Cellular, Wi-Fi & Medium & NAVTEQ LocationPoint Advertising \\
\hline $\begin{array}{l}\text { Environment } \\
\text { monitoring and } \\
\text { protection }\end{array}$ & $\begin{array}{l}\text { Transport of danger materials } \\
\text { Pollutants tracking }\end{array}$ & $\begin{array}{l}\text { GPS, } \\
\text { Barcodes, } \\
\text { RFID }\end{array}$ & $\begin{array}{l}\text { High to } \\
\text { meduim }\end{array}$ & $\begin{array}{l}\text { EcoSense } \\
\text { http://ecosense.au.dk/ }\end{array}$ \\
\hline $\begin{array}{l}\text { Wildlife (animal) } \\
\text { monitoring }\end{array}$ & Animal tracking and protection & $\begin{array}{l}\text { GPS, ARGOS, } \\
\text { sensor } \\
\text { networks }\end{array}$ & $\begin{array}{l}\text { Medium to } \\
\text { low }\end{array}$ & $\begin{array}{l}\text { ProjectNoah } \\
\text { (http://www.projectnoah.org/) }\end{array}$ \\
\hline
\end{tabular}

\section{Seamless Integration of Positioning Methods and Systems}

When considering the accuracy, availability and scope of a positioning system while reducing its power consumption and cost, the integration of several positioning technologies and systems is an option that might be relevant in many contexts. Such integration should also provide seamless handover of integrated positioning systems that selects the most suitable positioning 
continuously monitored, processed and analyzed at the server side. Continuous location data streams define trajectories of moving objects that provide unprecedented information to understand their mobility and behavior. Within this research direction, methods and algorithms of mobility data stream management are investigated, as well as application of high-performance and data-intensive computing techniques and systems for handling massive trajectory data collections, both on-line and offline, such as cluster/grid (MPI), cloud computing techniques (MapReduce) and GPGPU (OpenCL, CUDA).

\section{Participatory Location- Based Sensing}

With the proliferation of mobile phones with increasing sensing capabilities in everyday use, an important source of sensor data has become the users with their mobile devices. Thanks to an increasing number of built-in sensors: ambient light, orientation, accelerometer, sound, camera, velocity, GPS, but also usergenerated content (video, photo, sound, text, etc.), each mobile device can continuously capture, process, analyze and transmit spatially and temporally referenced data describing the context and the situation of the user. We need efficient methods, techniques, algorithms and systems that provide collection, monitoring, processing and analyzing of large volumes of moving sensor data that define context and situation relevant to a particular application domain (Predic et al., 2013).

\section{Semantic Location and Trajectory Data}

Once the location of a moving object is known, in either geometric or symbolic form, many other pieces of information can be inferred to enrich such location information. The research aims to develop methods and software tools to provide collection, processing and analysis of location and trajectory semantics. Semantic locations and trajectories enable development of advanced LBSs which should provide more intelligent, proactive and valuable services to users navigating in outdoor and indoor environments (Afyouni et al., 2012). Processing, analysis and mining of semantic locations/trajectories provide insights in semantics of movement and recognition of user activities, behavior and prediction of a future movement.

\section{Privacy in Location-Based Services}

Although location determination provides many valuable location-based applications and services to mobile users, revealing people's locations to potentially untrusted service providers poses significant privacy concerns. There is a trade-off between the quality of services offered by a LBS provider and the privacy of a user's location. The research in this direction focuses on the protection of sensitive locations against LBS providers and untrusted members of collaborative geo-social networks (Khoshgozaran \& Shahabi, 2010).

\section{CONCLUSION}

This article gives an overview of the methods, techniques, technologies and systems involved in the development of location-based services for mobile users and moving objects tracked in the environment. As no positioning system is accessible everywhere, compromises between accuracy, scope, latency, privacy and costs may result in a system that seamlessly integrate two or more positioning technologies and systems.

Research challenges in pervasive positioning cover not only data collection methods which are reaching a relatively mature level, but also semantic and technical research avenues where the objective will be to make the best possible use of the data. This is why mobile information systems are the object of several exciting research efforts, from semantic to participatory and privacy, and where several international projects are under development such as the EU projects MOVE (Knowledge Discovery from Moving Objects - http:// www.move-cost.info/) and MODAP (Mobility, Data Mining, and Privacy - http://www.modap.org/). No doubts that other research projects will soon emerge at the international level as opportunities cover many different location-based application domains that can be successfully applied to outdoor and indoor environments. 


\section{REFERENCES}

Afyouni, I., Ray, C., Ilarri, S., \& Claramunt, C. (2012). Algorithms for continuous location-dependent and context- aware queries in indoor environments, In I. Cruz (Eds.) In Proceedings of the 20th ACM SIGSPATIAL International Conference on Advances in GIS (pp. 329-338). ACM Press.

Bahl, P., \& Padmanabhan, V. N. (2000). Radar: an inbuilding RF-based user location and tracking system. In Proceedings of the INFOCOM (pp.775-784).

Barshan, B., \& Durrant-Whyte, H. F. (1995). Inertial navigation systems for mobile robots. IEEE Transactions on Instrumentation and Measurement, 44(1), 61-67. doi:10.1109/19.368102

Brumitt, B., Meyers, B., Krumm, J., Kern, A., \& Shafer, S. A. (2000). EasyLiving: Technologies for intelligent environments. In Proceedings of the $2^{\text {nd }}$ International Symposium on Handheld and Ubiquitous Computing (pp. 12-29). London, UK: Springer-Verlag.

Figueiras, J., \& Frattas, S. (2010). Mobile Positioning and Tracking: From Conventional to Cooperative Techniques. Wiley. doi:10.1002/9780470663035

Gu, Y., Lo, A., \& Niemegeers, I. (2009). A survey of indoor positioning systems for wireless personal networks. IEEE Communications Surveys \& Tutorials, 11(1), 13-32. doi:10.1109/SURV.2009.090103

Hightower, J., \& Borriello, G. (2001). Location systems for ubiquitous computing. IEEE Computer, 34(8), 57-66. doi:10.1109/2.940014

Hightower, J., Want, R., \& Borriello, G. (2000). SpotON: an indoor $3 D$ location sensing technology based on RF signal strength. CSE. Seattle, WA: University of Washington.

Khoshgozaran, A., \& Shahabi, C. (2010). A taxonomy of approaches to preserve location privacy in locationbased services. International Journal Computational Science and Engineering, 5(2), 86-96. doi:10.1504/ IJCSE.2010.036819
Laere, O. V., Strobbe, M., Leroux, P., Dhoedt, B., Turck, F. D., \& Demeester, P. (2008). Enabling Platform for Mobile Content Generation Based on 2D Barcodes. In H. R. Arabnia \& V. A. Clincy (Eds.), International Conference on Internet Computing (pp. 209-214). CSREA Press.

Liu, H., Darabi, H., Banerjee, P., \& Liu, J. (2007). Survey of wireless indoor positioning techniques and systems. IEEE Transactions on Systems, Man and Cybernetics. Part C, Applications and Reviews, 37(6), 1067-1080. doi:10.1109/TSMCC.2007.905750

Ni, L. M., Liu, Y., Lau, Y. C., \& Patil, A. P. (2004). Landmarc: indoor location sensing using active RFID. Wireless Networks, 10(6), 701-710. doi:10.1023/ B:WINE.0000044029.06344.dd

Predic, B., Yan,Z., Eberle, J., Stojanovic, D., \& Aberer, K. (2013).ExposureSense: Integrating Daily Activities with Air Quality using Mobile Participatory Sensing. In Proceedings of 11 th IEEE Pervasive Computing and Communication Conference (pp. 303-305).

Sahinoglu, Z., Gezici, S., \& Guvenc, I. (2008). Ultrawideband Positioning Systems: Theoretical Limits, Ranging Algorithms, and Protocols. Cambridge, UK: Cambridge University Press. doi:10.1017/ CBO9780511541056

Skog, I., \& Handel, P. (2009). In-car positioning and navigation technologies - a survey. IEEE Transactions on Intelligent Transportation Systems, 10(1), 4-21. doi:10.1109/TITS.2008.2011712

Want, R., Hopper, A., Ao, V. F., \& Gibbons, J. (1992). The active badge location system. ACM Transactions on Information Systems, 10(1), 91-102. doi:10.1145/128756.128759

\section{ADDITIONAL READING}

Ahson, S., \& Ilyas, M. (Eds.). (2010). Location-Based Services Handbook: Applications, Technologies, and Security. CRC Press. doi:10.1201/9781420071986 
Damiani, M. L., Silvestri, C., \& Bertino, E. (2011). Fine-grained cloaking of sensitive positions in location sharing applications. IEEE Pervasive Computing/ IEEE Computer Society [and] IEEE Communications Society, 10(4), 64-72. doi:10.1109/MPRV.2011.18

Giannotti, F., \& Pedreschi, D. (Eds.). (2008). Mobility, Data Mining, and Privacy. Springer. doi:10.1007/9783-540-75177-9

Grewal, M. S., Weill, L. R., \& Andrews, A. P. (2007). Global Positioning System, Inertial Navigation, and Integration (2nd ed.). Hoboken, NJ: John Wiley \& Sons. doi:10.1002/0470099720

Hansen, R., Wind, R., Jensen, C. S., \& Thomsen, B. (2009). Seamless indoor/outdoor positioning handover for location-based services in Streamspin. In Proceedings of Mobile Data Management (pp. 267-272). IEEE Computer Society. doi:10.1109/MDM.2009.39

Hazas, M., \& Ward, A. (2002). A novel broadband ultrasonic location system. In Proceedings of the UbiComp '02: 4th International Conference on Ubiquitous Computing (pp. 264-280). London, UK: Springer.

Hightower, J., Brumitt, B., \& Borriello, G. (2002). The Location Stack: A Layered Model for Location in Ubiquitous Computing. In Proceedings of the 4th Workshop Mobile Computing Systems and Applications (pp. 22-28). IEEE Computer Society.

Kolodziej, K. W., \& Hjelm, J. (2006). Local Positioning Systems: LBS Applications and Services. CRC Press, Taylor \& Francis Group, LLC.

Küpper, A. (2005). Location-Based Services: Fundamentals and Operation. West Sussex, UK: John Wiley \& Sons, Ltd. doi:10.1002/0470092335

Kushki, A., Plataniotis, K. N., \& Venetsanopoulos, A. (2012). WLAN Positioning Systems: Principles and Applications in Location-Based Services. Cambridge, UK: Cambridge University Press.

Lane, N. D., Miluzzo, E., Lu, H., Peebles, D., Choudhury, T., \& Campbell, A. T. (2010). A survey of mobile phone sensing. IEEE Communications Magazine, 48(9), 140-150. doi:10.1109/MCOM.2010.5560598

Mannings, R. (2008). Ubiquitous Positioning. Artech House Publishers.
Priyantha, N. B. (2005). The Cricket Indoor Location System. (PhD thesis).Cambridge, MA: MIT.

Roxin, A., Gaber, J., Wack, M., \& Nait-Sidi-Moh, A. (2007). Survey of wireless geolocation techniques. In Proceedings of IEEE Globecom Workshops (pp. 1-9).

Schiller, J., \& Voisard, A. (2004). Location-Based Services. San Francisco, CA: Morgan Kaufmann Publishers.

Zheng, Y., \& Zhou, X. (Eds.). (2011). Computing with Spatial Trajectories. Springer. doi:10.1007/9781-4614-1629-6

\section{KEY TERMS AND DEFINITIONS}

Angulation: Triangulation technique used to determine location of a mobile device by measuring angles to it from transmitters at known locations.

Geometric Location: Physical positional information that include a set of coordinates, e.g., for a GPS receiver it might be latitude, longitude and height, in a particular reference system.

Hybrid Positioning: Integration of different location sensing systems and technologies that can be advantageous by increasing accuracy, availability and precision beyond that could be obtained using single systems.

Proximity: Proximity of an object to a sensor can be determined either by direct contact or by a nearness measurement within certain threshold.

Symbolic: Location: Qualitative positional information, e.g., identifier of a room or point of interest where a target object is located or the area in which it is located.

Triangulation: Location sensing techniques that use the geometry of triangle and the measurement of its distances and angles to compute location of a target object.

Trilateration: Triangulation technique used to determine a location by determining the intersection of hyperbolas derived from the range measurements between transmitters and a mobile device. 